\title{
Benign and low-grade fibroepithelial neoplasms of the breast have low recurrence rate after positive surgical margins
}

\author{
Morgan L Cowan ${ }^{1}$, Pedram Argani ${ }^{1,2}$ and Ashley Cimino-Mathews ${ }^{1,2}$ \\ ${ }^{1}$ Department of Pathology, The Johns Hopkins Hospital, Baltimore, MD, USA and ${ }^{2}$ Department of Oncology, \\ The Johns Hopkins Hospital, Baltimore, MD, USA
}

\begin{abstract}
Breast phyllodes tumors are uncommon fibroepithelial neoplasms with a range of histologic features. Surgical excision is the primary management, but the need for excision to negative margins in benign and borderline phyllodes tumors is unclear. Here, we review the surgical management patterns and outcomes of 90 patients with benign and low-grade fibroepithelial lesions of the breast treated at our institution, including 19 borderline phyllodes tumors, 52 benign phyllodes tumors, and 19 representative neoplasms with overlapping features of fibroadenoma and benign phyllodes tumors, which were classified as 'fibroadenomas with phyllodal features'. In total, $52(58 \%)$ had positive surgical margins on first excision, and of these $17(33 \%)$ underwent re-excision to achieve negative margins. Residual tumor was identified in three (18\%) re-excisions. Patients with fibroadenoma with phyllodal features were more likely to have a positive surgical margin than with benign phyllodes tumors or borderline phyllodes tumors ( 89 vs $49 \%, P=0.0015$ ), and were less likely to undergo re-excision for positive margins (12 vs $43 \%, P=0.031)$. In total, there were three recurrences $(3 \%)$, with one per fibroadenoma with phyllodal features, benign phyllodes tumor, and borderline phyllodes tumor. There was no statistically significant difference in recurrence rates between patients with positive or negative margins, or between patients with positive margin with or without re-excision. The extent of the positive margin did not predict recurrence. In conclusion, the recurrence rate of benign and low-grade fibroepithelial lesions is low and not associated with the original margin status. Patients with fibroadenomas with phyllodal features, benign phyllodes tumors, or selected borderline phyllodes tumors and positive margins on initial excision may be managed conservatively, with close follow-up and timely re-excision of any potential recurrence.
\end{abstract}

Modern Pathology (2016) 29, 259-265; doi:10.1038/modpathol.2015.157; published online 8 January 2016

Breast phyllodes tumors are relatively rare fibroepithelial neoplasms that primarily affect middle-aged women and have variable risk of local recurrence and metastasis. The WHO categorizes phyllodes tumors as benign, borderline, and malignant based on a constellation of features, including the degree of stromal hypercellularity and atypia, stromal mitotic rate, the presence of stromal overgrowth, tumor circumscription, and the presence of malignant heterologous elements. ${ }^{1}$ Benign and borderline phyllodes tumors carry the

Correspondence: Dr A Cimino-Mathews, MD, Department of Pathology, The Johns Hopkins Hospital, 401 North Broadway Street, Weinberg 2242, Baltimore, MD 21231, USA.

E-mail: acimino@jhmi.edu

This study was presented at the United States and Canadian Academy of Pathology Annual Meeting, 2014.

Received 9 September 2015; revised 18 November 2015; accepted 20 November 2015; published online 8 January 2016 potential for local recurrence, with the risk of metastasis primarily limited to malignant phyllodes tumors. $^{2-5}$ Precise classification of these benign and low-grade fibroepithelial neoplasms can be difficult in daily practice. The morphologic features of fibroadenomas with pronounced intracanalicular growth pattern and of benign phyllodes tumors overlap, leading to high interobserver variability in classification of these lesions, even among breast pathology experts. ${ }^{6}$ The WHO recognizes the presence of morphologic overlap and suggests the term 'benign fibroepithelial neoplasms' with an explanation as to why this term is used in situations where precise classification is difficult. ${ }^{1}$ At our institution, we use the term 'fibroadenoma with phyllodal features' for these lesions (see Materials and methods).

Regardless of tumor grade, surgery is the main treatment for patients with fibroepithelial neoplasms. Complete excision may be difficult, although 
Table 1 Clinicopathologic characteristics of patients with benign and low-grade fibroepithelial neoplasms

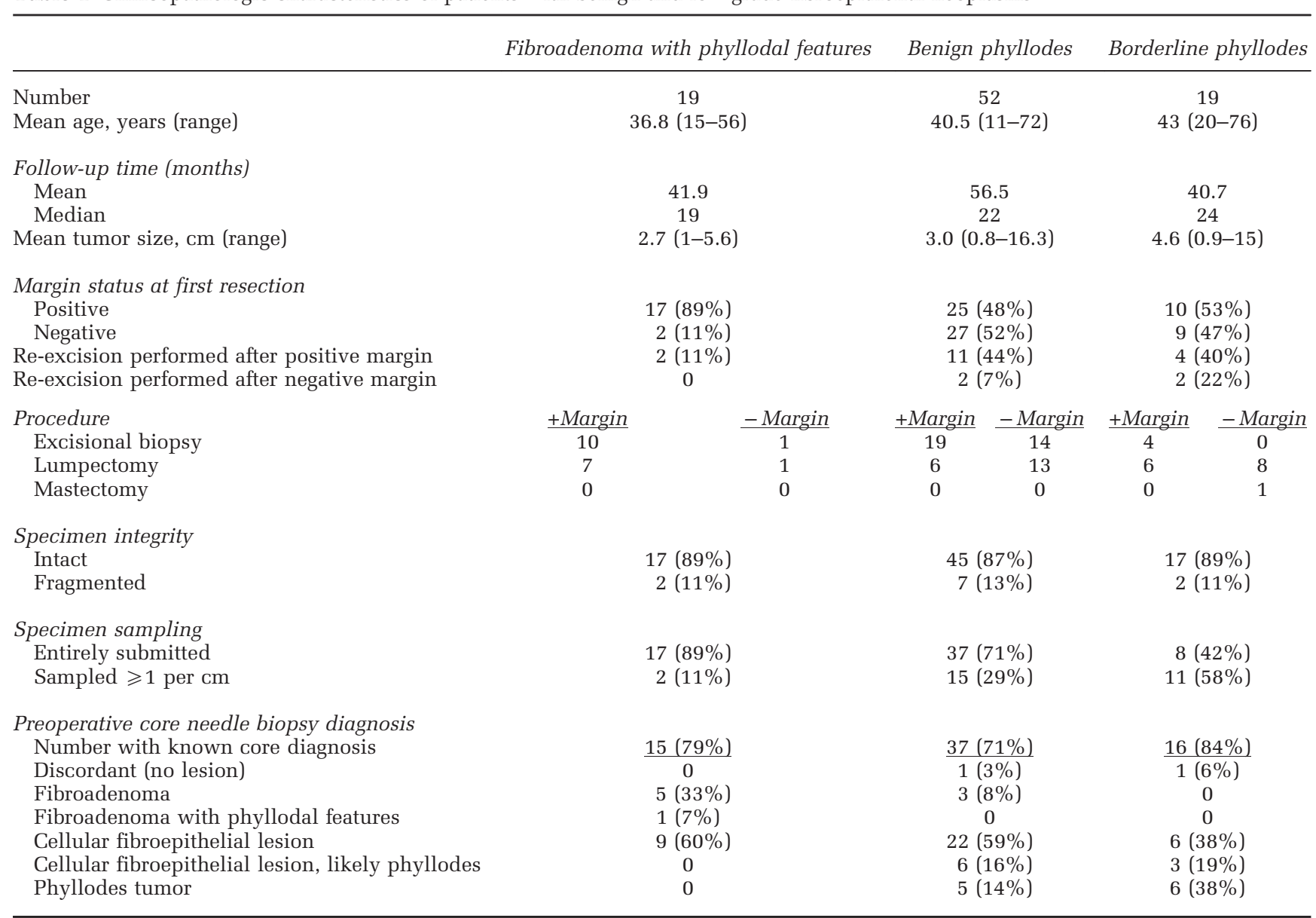

Abbreviations: +margin, positive margin; - margin, negative margin.

Underlined values signify that the following values were \% of the number of cases with known biopsy results.

re-excision to negative margins has been recommended. ${ }^{7}$ In practice, the surgical management of positive margins after primary excision in patients with these low-grade fibroepithelial neoplasms is variable, and not all patients undergo re-excision. We hypothesize that the recommendation to excise phyllodes tumors to negative margins results in overtreatment of the subset of patients with benign phyllodes tumors. In this study, we investigated the margin status, re-excision rate, and recurrence rate in patients with low-grade fibroepithelial neoplasms of the breast, including fibroadenoma with phyllodal features, benign phyllodes tumors, and borderline phyllodes tumors, to better characterize the surgical management patterns and clinical behavior of these tumors.

\section{Materials and methods}

\section{Case Identification and Review}

This study was approved by the Institutional Review Boards of our institution. Pathology archives were searched for patients diagnosed with 'benign phyllodes tumor' and 'borderline phyllodes tumor'. To capture older cases with previous nomenclature that would now be termed borderline phyllodes tumor, we also included the term 'low grade malignant phyllodes tumor'. We included all patients who underwent excision of their tumor at our institution from 1989 to 2013. In addition, a consecutive series of patients whose tumors had features intermediate between benign phyllodes tumor and fibroadenoma, corresponding to the WHO descriptor of 'benign fibroepithelial neoplasm' and diagnosed as 'fibroadenoma with phyllodal features,' were included for comparison. Clinicopathologic data including patient age, preoperative core needle biopsy diagnosis, tumor diagnosis on excision, tumor size, the margin status as reported, initial and subsequent surgical procedures, follow-up time, subsequent recurrence, and treatments received were recorded. For the purposes of this study, the surgical margin was considered positive only if the tumor was present at the inked margin of resection. All available slides were retrieved from our archives and re-examined to confirm the diagnosis, note the distance to the nearest margin, and measure the extent of a positive margin when present. 

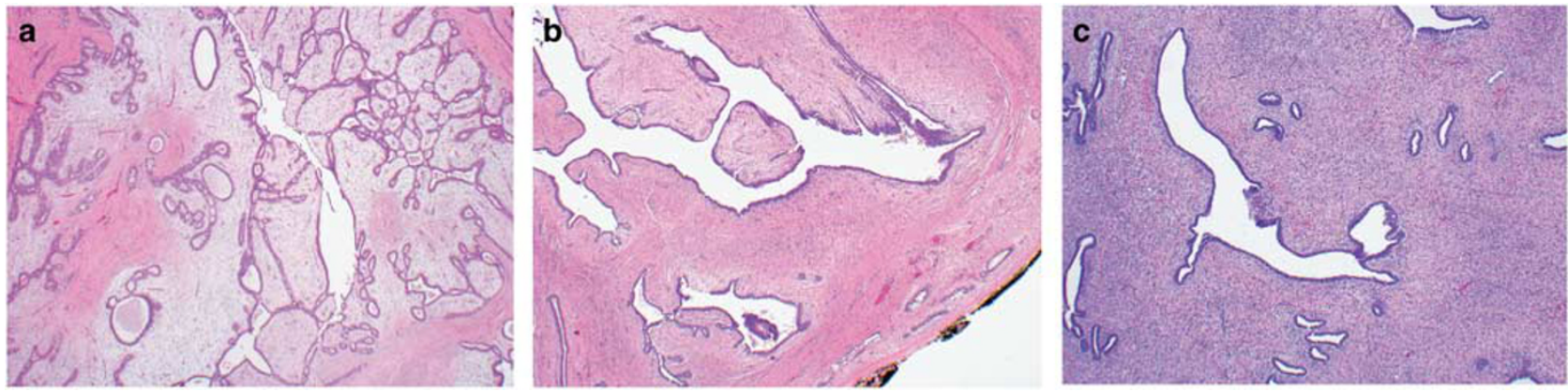

Figure 1 Histologic features of benign and low-grade fibroepithelial lesions of the breast. A fibroadenoma with phyllodal features displays mildly cellular stroma with a focally prominent intracanalicular and leaf-like architecture $(\mathbf{a}, \times 20)$. A benign phyllodes tumor displays a mildly hypercellular stroma with no atypia, a wide-spread prominent leaf-like architecture, a circumscribed border, and a positive margin in this field $(\mathbf{b}, \times 20)$. A borderline phyllodes tumor displays increased stromal cellularity with mild cytologic atypia $(\mathbf{c}, \times 20)$.

\section{Statistical Analysis}

The number of patients with initial positive and negative margins, subsequent re-excision, and tumor recurrence were compared using the Fisher exact test.

\section{Results}

\section{Clinicopathologic Characteristics}

The clinicopathologic features are summarized in Table 1. In total, 94 cases were initially identified, and slides were available for review in 79 (84\%) cases. Four cases with a diagnosis of 'low grade malignant phyllodes tumor' were discarded after slide review due to one or more features of a more aggressive tumor: high mitotic rate ( $>10$ mitoses per 10 high power fields), stromal overgrowth, or extensive infiltrative growth pattern. A total of 90 cases were then included for study, consisting of 52 benign phyllodes tumors, 19 borderline phyllodes tumors, and a representative sample of 19 fibroadenoma with phyllodal features (Figure 1). There were no follow-up data available for 13 patients. The mean follow-up interval for all patients with at least 1 month of follow-up was 57.7 months, with a median interval of 40.5 months (range 1-282 months). The mean age at the time of excision was 40.3 years, with a median age of 43 years (range 11-76 years). There was no significant difference in mean age or mean follow-up time in patients with fibroadenoma with phyllodal features, benign phyllodes tumors, or borderline phyllodes tumors.

All but one patient were initially treated with either an excisional biopsy or a lumpectomy; the remaining one patient with a borderline phyllodes tumors underwent a mastectomy. More patients with fibroadenoma with phyllodal features and benign phyllodes tumors had excisional biopsies compared to patients with borderline phyllodes tumors (62\% fibroadenoma with phyllodal features and benign phyllodes tumors compared to $21 \%$ borderline phyllodes tumors; $P=0.0018$ ). The majority (88\%) of gross specimens were received intact, with a small proportion $(12 \%)$ received as two or more tissue fragments. The mean tumor size of the fibroadenoma with phyllodal features, benign phyllodes tumors, and borderline phyllodes tumors was 2.7, 3.0, and $4.6 \mathrm{~cm}$, respectively, with the borderline phyllodes tumors significantly larger than the fibroadenoma with phyllodal features $(P=0.04)$. The majority $(69 \%)$ of specimens were entirely submitted, and the remaining $31 \%$ were extensively sampled with at least one section per centimeter, if not greater. More fibroadenoma with phyllodal features were entirely submitted (89\%) compared to benign phyllodes tumors $(71 \%)$ or borderline phyllodes tumors ( $42 \% ; P=0.04$ and 0.02 , respectively), likely reflecting the higher proportion of excisional biopsy specimens and smaller tumor size in the fibroadenoma with phyllodal features specimens.

The diagnostic report from a preceding core needle biopsy was available for the majority $(76 \%)$ of patients (Table 1). Of these, a core biopsy diagnosis of fibroadenoma was made more often in patients with fibroadenoma with phyllodal features on excision than benign phyllodes tumors or borderline phyllodes tumors (33 vs $8 \%$ and $0 \%, P=0.01$ ). The three patients with a diagnosis of fibroadenoma on core biopsy and benign phyllodes tumors on excision were recommended for surgical resection due to either continued growth of the lesion $(n=2)$ or history of multiple fibroepithelial lesions $(n=1)$. A core needle biopsy diagnosis of 'cellular fibroepithelial lesion' (with the recommendation to defer to excision for final classification) was the most common core needle biopsy diagnosis in all three subgroups, specifically in $60 \%$ patients with fibroadenoma with phyllodal features, $59 \%$ patients with benign phyllodes tumors, and $38 \%$ patients with borderline phyllodes tumors $(P=$ n.s.). A core needle biopsy diagnosis of 'cellular fibroepithelial lesion, strongly suspect phyllodes tumor' or a specific diagnosis of 'phyllodes tumor' was made more often in patients with benign phyllodes tumors or borderline phyllodes tumors on excision (30 and $56 \%$ ), compared to no patient with fibroadenoma 


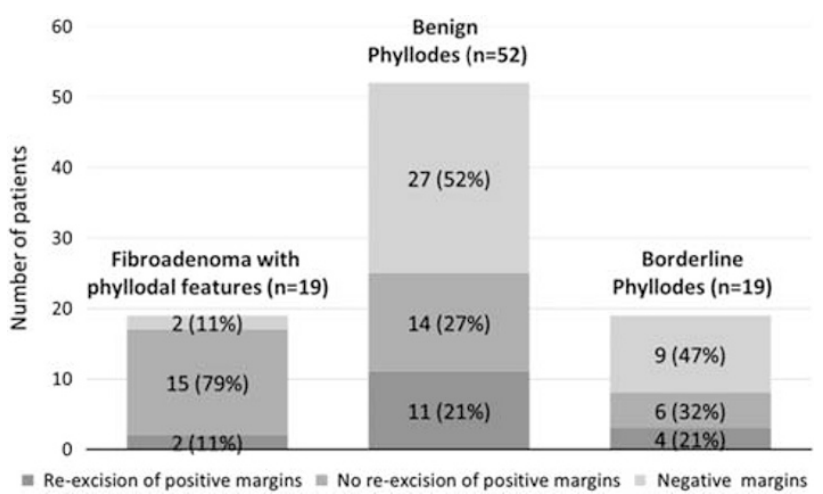

Figure 2 Rates of margin positivity and margin re-excision across benign and low-grade fibroepithelial lesions of the breast. The graph shows the absolute number of patients in each diagnostic category (fibroadenoma with phyllodal features, benign phyllodes and borderline phyllodes by margin status, and subsequent management of positive margin. Percentages were calculated using the total number of patients in a specific diagnostic category.

with phyllodal features on excision $(P=0.003)$. A core needle biopsy diagnosis of 'phyllodes tumor' was made more often in patients with borderline phyllodes tumors on excision (38\%) than benign phyllodes tumors $(14 \%, P=0.07)$.

\section{Positive Margin Status and Re-Excision Rate}

Overall, $52(58 \%)$ patients had positive surgical margins with tumor present at the inked tissue edge of the initial resection. Patients with excisional biopsy specimens were more likely to have positive margins than those with lumpectomies (69\% compared to $46 \%, P=0.05)$. Of patients with positive margins, $17(33 \%)$ patients underwent additional surgery for margin re-excision, and residual tumor was identified in only three $(18 \%)$ re-excisions. These three patients were all initially diagnosed with benign phyllodes tumor, with residual benign phyllodes tumor in each re-excision; the final resection margin was negative in all three cases.

When separated by tumor type (Table 1 and Figure 2), the patients diagnosed with fibroadenoma with phyllodal features were more likely to have a positive surgical margin than patients with benign phyllodes tumors or borderline phyllodes tumors (89 vs $49 \%, P=0.0015$ ), and patients with fibroadenoma with phyllodal features were less likely to undergo re-excision for positive margins $(12 \%)$ compared to patients benign phyllodes tumors or borderline phyllodes tumors $(43 \% ; P=0.031)$. Specifically, 17 $(89 \%)$ patients with fibroadenoma with phyllodal features had positive surgical margins after the first excision, and two (12\%) of those underwent re-excision, with no residual tumor identified. In total, $25(48 \%)$ patients with benign phyllodes tumors had positive surgical margins, and 11 (44\%) of these patients underwent re-excision. Residual tumor was identified in three $(27 \%)$ of the benign

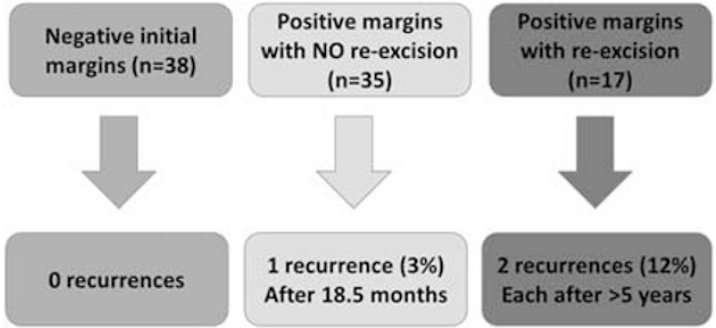

Figure 3 Recurrence rates of benign and low-grade fibroepithelial lesions of the breast by surgical margin status at primary excision. This diagram shows the number of recurrences grouped by initial margin status and subsequent surgical treatment (negative margin, positive margin with re-excision, and positive margin without reexcision). Percentages are calculated based on the total in each management group.

phyllodes tumors re-excision specimens. A total of $10(53 \%)$ patients with borderline phyllodes tumors had positive initial surgical margins, and four (40\%) of those underwent re-excision with no residual tumor identified.

\section{'Close' Margins}

Slides were available for review in 31 patients with negative resection margins. Tumor was present $<3 \mathrm{~mm}$ from the ink in 24 cases and was located $\geq 3 \mathrm{~mm}$ from ink in the remaining six cases. Tumor was present $\leq 1 \mathrm{~mm}$ from ink in 17 cases. Surgical margin re-excision was also performed in 4 (11\%) patients with negative margins (ie, without tumor present at ink). Two of these patients had benign phyllodes tumors with tumor present $<1 \mathrm{~mm}$ from ink, and two of these patients had borderline phyllodes tumors with tumor present $<1$ and $2 \mathrm{~mm}$ from ink, respectively. No residual tumor was identified in any of the re-excision specimens.

\section{Recurrences}

Of the 90 patients included in this study, there were three reported recurrences (3\%), with one recurrence each per diagnostic category (fibroadenoma with phyllodal features, benign phyllodes tumors, and borderline phyllodes tumors). All three recurrences occurred in patients who had initial positive surgical margins, but two of these patients had re-excision to negative margins (Figure 3). Thus, recurrences occurred in $3 \%(1 / 35)$ of patients with final positive margins and in $4 \%(2 / 55)$ of patients with final negative margins $(P=1.0)$. One of the recurrences occurred in a patient who did not undergo a re-excision after the initial positive margin. This patient was initially diagnosed with fibroadenoma with phyllodal features, and after 18 months presented with a tumor in the lumpectomy site that was presumed recurrent and diagnosed as a benign phyllodes tumor. At the time of re-presentation with this recurrence, she was also found to have a 
concurrent contralateral benign phyllodes tumor. The other two recurrences were in patients who did undergo re-excision to negative final surgical margins. Neither re-excision specimen contained additional tumor. One of these two recurrences was a benign phyllodes tumor, which recurred 65 months later as a benign phyllodes tumor; the association of the recurrence with the previous lumpectomy site was unknown. The other was a borderline phyllodes tumors, which recurred 5 years later as a malignant phyllodes tumor, in the same quadrant as the original tumor. This patient developed metastases and ultimately died of disease.

In patients with initial positive margins (Figure 3), the difference in recurrence rate between the patients who underwent re-excision to negative final surgical margins (12\%) and those who did not undergo re-excision (3\%) is not statistically significant $(P=0.25)$. In addition, there was no significant difference in recurrence rate between patients with negative initial margins and those with positive margins who did not undergo re-excision to negative final margins ( 0 vs $3 \%, P=0.48$ ), nor was there a difference in recurrence rate between patients with negative initial margins and those with positive initial margins who did undergo re-excision to negative final margins (0 vs $12 \%, P=0.09$ ).

\section{Length of Positive Margin and Recurrence Rate}

Of the 44 patients with positive margins and slides available for review, $28(64 \%)$ patients had a positive margin length $\geq 5 \mathrm{~mm}$, and $16(36 \%)$ patients had a positive margin length $<5 \mathrm{~mm}$. In patients with initial positive margins, the extent of the positive margin was not significant in predicting recurrence. Although the three patients with recurrence tumors all had initial positive margins of $>5 \mathrm{~mm}$, there were 24 patients with positive margins of $>5 \mathrm{~mm}$ who did not recur.

\section{Discussion}

Classification of breast fibroepithelial lesions can be diagnostically challenging due to morphologic overlap between the subtypes, particularly at the low end of the spectrum where there can be substantial similarity between cellular fibroadenoma and benign phyllodes tumors. ${ }^{6}$ The current recommended treatment for fibroepithelial neoplasms is surgical excision with negative margins, although some investigators advocate a more aggressive management such as radiation therapy in phyllodes tumors. ${ }^{8}$ Surgical recommendations are based on a clear and reproducible inverse association between widely clear margin status and recurrence rate for malignant phyllodes tumors. ${ }^{9-13}$ The definition of negative margin varies widely across authors, with some advocating a clear margin of $>1 \mathrm{~cm} .{ }^{14,15}$ Historically, incomplete excision of any phyllodes tumors has been correlated with local recurrences. ${ }^{3,9,16-21}$ However, many studies correlating positive or 'close' surgical margins with an increased risk of local recurrence do not separate benign or borderline phyllodes tumors from high grade, malignant phyllodes tumors. ${ }^{16,17,20}$

In contrast, other studies have failed to demonstrate a direct relationship between margin status or width of negative margins and recurrence. In a series of 285 phyllodes tumors, margin status was not associated with risk of local recurrence. ${ }^{22}$ In a study of 164 phyllodes tumors, a 1-cm wide margin showed no advantage over a narrower negative margin. ${ }^{20}$ In a smaller single institution studies of 33 (ref. 23) and 44 (ref. 24) phyllodes tumors, there was no relationship between surgical margin width ${ }^{23}$ or margin status ${ }^{24}$ and local disease recurrence. Additional series have also documented recurrences in phyllodes tumors that were originally excised to negative margins. ${ }^{15,17}$ This may reflect multifocality of tumors, or the presence of associated 'fibroadenomatoid change' in the surrounding breast tissue. ${ }^{21}$ Some authors have advocated a watchful waiting for patients with benign and low-grade tumors and positive surgical margins. ${ }^{13,25}$ One potential downside to immediate re-excision is cosmetic; if another lesion that needs excision subsequently appears, a third resection would be necessary.

Here, we present a retrospective analysis of the margin status and surgical management patterns for 90 patients with benign and low-grade fibroepithelial neoplasms of the breast. Although the retrospective study design is a limitation, the rarity of these tumors makes a prospective design difficult. The strengths of this study include a relatively large sample size $(n=90)$ with pathologic re-review of all available slides, including measurement of distance to margin and length of positive margins, as well as inclusion of both fibroadenomas with phyllodal features and benign phyllodes tumors with overlapping features.

In the entire cohort, the recurrence rate was very low $(3 \%)$. A large fraction $(55 \%)$ of patients had positive surgical margins on first excision, but neither the presence of nor the extent of the positive margin ( $>$ or $<5 \mathrm{~mm}$ in length) was significant in predicting recurrence. Of these patients with positive margins, only one-third underwent re-excision. All re-excisions resulted in negative margins, but did not result in a statistically significant difference in recurrence rate compared to patients who did not have further surgery. Surprisingly, two of the three 'recurrences' in fact occurred in patients who were re-excised to negative margins. There was no difference in recurrence rates between fibroadenoma with phyllodal features and benign phyllodes tumors, affirming that it is reasonable to use the term 'benign fibroepithelial neoplasm' to encompass both entities.

Most phyllodes tumors recur within 1-3 years from diagnosis. ${ }^{10,13,16,21,25,26}$ Although some (13\%) patients had no follow-up, the remaining patients had a median follow-up of 40.5 months, which is 
beyond the reported 12-36 month average time to recurrence. In addition, two of the three recurrences in our series occurred after 5 years. After such a long time interval, it is possible that these tumors represented new primary lesions. The third recurrence occurred in a young patient who represented with bilateral disease after only 18 months. With three tumors within a 2-year period, this patient appears to have some underlying propensity for fibroepithelial neoplasms, and her ipsilateral 'recurrence' could also be a new primary lesion.

Also notable, while patients with fibroadenoma with phyllodal features were more likely to have positive margins on initial excision than those with benign phyllodes tumors or borderline phyllodes tumors ( 89 vs $48 \%$ and $42 \%$ ), they were much less likely to be taken back for re-excision (12 vs 44\% and $38 \%$ ). These findings are perhaps not surprising and suggest different surgical management patterns for patients with fibroadenoma with phyllodal features than phyllodes tumors, such that the surgeon may have been more likely to try for a 'wider' initial excision for a preoperative diagnosis of a phyllodes tumor than for a fibroadenoma. Indeed, the preoperative, core needle biopsy diagnoses likely impacted the surgical management decisions, such that more patients with fibroadenoma with phyllodal features on excision had a diagnosis of 'fibroadenoma' on core needle biopsy, and more patients with benign or borderline phyllodes tumors on excision had diagnoses of 'phyllodes tumor' or 'cellular fibroepithelial lesion, strongly suspect phyllodes tumor' on biopsy. The findings suggest that surgeons are already treating fibroadenoma with phyllodal features differently, as fewer of these patients with positive margins were re-excised.

About a third of patients with positive margins in our series underwent re-excision, and residual tumor was only present in the minority $(19 \%)$ of the re-excision specimens. This is in keeping with a previous study that found residual phyllodes tumor in only $16 \%$ of re-excision specimens performed for positive margins. ${ }^{15}$ Such low rates of residual disease combined with absence of statistically significant reduction in recurrence rate for patients with re-excision suggests that re-excision of positive margins in low-grade fibroepithelial neoplasms is unnecessary in the absence of gross residual disease. Importantly, this is a study limited to fibroadenoma with phyllodal features, benign phyllodes tumors, and borderline phyllodes tumors, and as such our findings do not apply to frankly malignant phyllodes tumors or those tumors at the 'upper end' of borderline with marked atypical features that fall just short of a diagnosis of malignant phyllodes tumor. Caution may be warranted in generalizing these recommendations to all patients with a diagnosis of borderline phyllodes tumor, due to both the lower number of borderline tumors included and the potential for interobserver variability in the classification between some borderline phyllodes tumors and malignant phyllodes tumors. Caution is also warranted in the rare cases in which the tumor is grossly transected, as opposed to microscopically involving the inked margin, as such tumors were not the subject of this study.

In conclusion, our findings suggest that patients with low-grade fibroepithelial lesions of the breast have low risk of local recurrence irrespective of margin status. Most patients may be conservatively and adequately managed by close clinical follow-up with timely re-excision of any subsequent recurrent lesion. Additional studies are warranted to clearly delineate an optimal follow-up algorithm, but beginning with clinicoradiographic reevaluation at 6 months followed by yearly evaluation may be a reasonable practice suggestion. Patients who undergo re-excision to obtain negative margins also need close clinical follow-up, as they too may develop subsequent recurrences.

\section{Disclosure/conflict of interest}

The authors declare no conflict of interest.

\section{References}

1 Tan PH, Tse G, Lee A et al. Fibroepithelial lesions. In: Lakhani SR, Ellis IO, Schnitt SJ, Tan PH, van de Vijver MJ (eds). WHO Classification of Tumours of the Breast. IARC: Lyon, 2012, pp 142-147.

2 Chaney AW, Pollack A, McNeese MD et al. Primary treatment of cystosarcoma phyllodes of the breast. Cancer 2000;89:1502-1511.

3 Tan PH, Jayabaskar T, Chuah KL et al. Phyllodes tumors of the breast: the role of pathologic parameters. Am J Clin Pathol 2005;123:529-540.

4 Reinfuss M, Mitus J, Duda K et al. The treatment and prognosis of patients with phyllodes tumor of the breast: an analysis of 170 cases. Cancer 1996;77: 910-916.

5 Lenhard MS, Kahlert S, Himsl I et al. Phyllodes tumour of the breast: clinical follow-up of 33 cases of this rare disease. Eur J Obstet Gynecol Reprod Biol 2008;138: 217-221.

6 Lawton TJ, Acs G, Argani $\mathrm{P}$ et al. Interobserver variability by pathologists in the distinction between cellular fibroadenomas and phyllodes tumors. Int J Surg Pathol 2014;22:695-698.

7 Mangi AA, Smith BL, Gadd MA et al. Surgical management of phyllodes tumors. Arch Surg 1999;134: 487-492.

8 Barth RJ Jr, Wells WA, Mitchell SE et al. A prospective, multi-institutional study of adjuvant radiotherapy after resection of malignant phyllodes tumors. Ann Surg Oncol 2009;16:2288-2294.

9 Ben Hassouna J, Damak T, Gamoudi A et al. Phyllodes tumors of the breast: a case series of 106 patients. Am J Surg 2006;192:141-147.

10 Kapiris I, Nasiri N, A'Hern R et al. Outcome and predictive factors of local recurrence and distant metastases following primary surgical treatment of 
high-grade malignant phyllodes tumours of the breast. Eur J Surg Oncol 2001;27:723-730.

11 Spitaleri G, Toesca A, Botteri E et al. Breast phyllodes tumor: a review of literature and a single center retrospective series analysis. Crit Rev Oncol Hematol 2013;88:427-436.

12 de Roos WK, Kaye P, Dent DM. Factors leading to local recurrence or death after surgical resection of phyllodes tumours of the breast. Br J Surg 1999;86:396-399.

13 Zurrida S, Bartoli C, Galimberti V et al. Which therapy for unexpected phyllode tumour of the breast? Eur J Cancer 1992;28:654-657.

14 Khosravi-Shahi P. Management of non metastatic phyllodes tumors of the breast: review of the literature. Surg Oncol 2011;20:e143-e148.

15 Guillot E, Couturaud B, Reyal F et al. Management of phyllodes breast tumors. Breast J 2011;17:129-137.

16 Taira N, Takabatake D, Aogi K et al. Phyllodes tumor of the breast: stromal overgrowth and histological classification are useful prognosis-predictive factors for local recurrence in patients with a positive surgical margin. Jpn J Clin Oncol 2007;37:730-736.

17 Chen WH, Cheng SP, Tzen CY et al. Surgical treatment of phyllodes tumors of the breast: retrospective review of 172 cases. J Surg Oncol 2005;91:185-194.

18 Moffat CJ, Pinder SE, Dixon AR et al. Phyllodes tumours of the breast: a clinicopathological review of thirty-two cases. Histopathology 1995;27:205-218.
19 Cheng SP, Chang YC, Liu TP et al. Phyllodes tumor of the breast: the challenge persists. World J Surg 2006;30: 1414-1421.

20 Jang JH, Choi MY, Lee SK et al. Clinicopathologic risk factors for the local recurrence of phyllodes tumors of the breast. Ann Surg Oncol 2012;19:2612-2617.

21 Barrio AV, Clark BD, Goldberg JI et al. Clinicopathologic features and long-term outcomes of 293 phyllodes tumors of the breast. Ann Surg Oncol 2007;14:2961-2970.

22 Yom CK, Han W, Kim SW et al. Reappraisal of conventional risk stratification for local recurrence based on clinical outcomes in 285 resected phyllodes tumors of the breast. Ann Surg Oncol 2015;22:2912-2918.

23 Lin CC, Chang HW, Lin CY et al. The clinical features and prognosis of phyllodes tumors: a single institution experience in Taiwan. Int J Clin Oncol 2013;18: 614-620.

24 Teo JY, Cheong CS, Wong CY. Low local recurrence rates in young Asian patients with phyllodes tumours: less is more. ANZ J Surg 2012;82:325-328.

25 Tan EY, Tan PH, Yong WS et al. Recurrent phyllodes tumours of the breast: pathological features and clinical implications. ANZ J Surg 2006;76:476-480.

26 Wei J, Tan YT, Cai YC et al. Predictive factors for the local recurrence and distant metastasis of phyllodes tumors of the breast: a retrospective analysis of 192 cases at a single center. Chin J Cancer 2014;33: $492-500$. 